\title{
COMMENTARY
}

\section{Measuring and reporting glycemic control in clinical trials: building a path to consensus}

\author{
Carmen L Soto-Rivera' and Michael SD Agus*2 \\ See related review by Finfer et al., http://ccforum.com/content/17/3/229
}

\begin{abstract}
Clinical trials over time have used a variety of approaches for both measuring tight glycemic control and reporting results. The review by Finfer and colleagues in this issue of Critical Care is a step toward consensus within the research community to standardize the way blood glucose is measured and reported in clinical trials. The authors propose using specific measures of central tendency and dispersion for reporting glucose, advocate the use of blood gas analyzers and elimination of point-ofcare glucose monitors in the intensive care unit, and comment on performance of continuous glucose monitors. As we await the release of updated rules from the International Standards Organization and process the new rules from the Clinical Laboratory and Standards Institute to regulate glucose monitoring, these recommendations should trigger many more conversations within the field as we strive for uniformity. However, we need to be cautious in prematurely proposing and adopting standards of care that fail to account for newer technology and data in this rapidly growing area of research.
\end{abstract}

Although whether to perform tight glycemic control (TGC) in the adult intensive care unit (ICU) continues to be debated among intensivists and endocrinologists worldwide, how to report and assess TGC took a constructive step forward with the publication of the consensus recommendations in this issue of Critical Care [1]. The authors, some of whom are the principal investigators from many of the major adult TGC trials in

*Correspondence: michael.agus@childrens.harvard.edu

${ }^{2}$ Division of Medicine Critical Care, Department of Medicine, Boston Children's

Hospital; Department of Pediatrics, Harvard Medical School, 300 Longwood

Avenue, Boston, MA 02115, USA

Full list of author information is available at the end of the article the field, present a compelling case for uniformity in assessment and reporting of future trials.

The group discussed and made recommendations in five areas: (a) measurement of intermittent blood glucose (BG) and characterization of those measurements, (b) performance standards for BG measurement devices, (c) use of continuous glucose monitoring (CGM), (d) assessment of CGM, and (e) performance of CGM. Although they cited much of the relevant published literature over the last several years, their task was daunting, particularly with new and improved BG measurement technology just recently brought to the inpatient setting [2]. The authors ultimately produced consensus recommendations, which are excellent first steps toward the laudable goal of uniform assessment and reporting.

In considering adoption of new standards, one has to take into account the quality and quantity of evidence that supports them as well as the structure of the formal analysis of the data. Finfer and colleagues put forth many resolutions to open questions. If adopted by clinical researchers, these standards would allow the signal to be noticed above the noise. However, some of the 'noise' that exists in the TGC literature represents reasonable variability in experimental approaches to performing and measuring TGC as well as in real-world implementation. For example, the authors acknowledge that protocols that increase BG sampling frequency as BG drops below a certain range will have a lower BG mean. However, the meeting consensus stipulates that the central tendency be reported as a median of patients' individual means, which does not mitigate the acknowledged bias.

When stipulating methods of measuring BG at the bedside in the ICU, the authors review performance of hospital meters from the prior generation of technology and rightly conclude that the meters are inadequate for ICU monitoring. But with a new generation of hospital BG meters on the market, new ICU evaluations are surely warranted [2]. Similarly, the cited International Standards Organization (ISO) and Clinical Laboratory and Standards Institute (CLSI) rules for BG meters are inappropriately generous for ICU use [3,4]; however, new rules have been developed by both organizations and are 
currently being rolled out [5,6]. Although blood gas analyzers are widely accepted to have outstanding precision and accuracy, they are not present in every ICU. Stipulation in a consensus document that glucose meters are unacceptable on the basis of prior generations of devices might discourage investigators from conducting clinical trials. In fact, the largest adult and pediatric trials published to date used this prior generation of devices with relatively low rates of severe hypoglycemia (7\% and $3 \%$ ), although the pediatric trial also used a computerized algorithm augmented with CGM $[7,8]$. The authors make similarly strong statements about the use of CGM in TGC, concluding that there are insufficient data about performance in the ICU.

Finfer and colleagues provide a first attempt at addressing the major issues in the field of conducting TGC in a clinical trial. The authors take a definitive position on a host of challenges facing TGC investigators, allowing the field to move forward. The phrase 'the devil is in the details' was not coined to describe clinical research, but those of us engaged in its conception and design know that it may as well have been. In trying to pin down many of those details, the authors are to be congratulated. However, it behooves all of us in the field to continue to advance and refine the conversation and to update our conclusions with the most recently available devices and data so that, ultimately, in both adult and pediatric critical care, we can discern which populations derive what benefit when a well-defined therapy is applied to their care.

Abbreviations

$\mathrm{BG}$, blood glucose; CGM, continuous glucose monitoring; ICU, intensive care unit; TGC, tight glycemic control.

\section{Competing interests}

MA is a consultant to Roche Diagnostics (Basel, Switzerland) on their hospitalbased glucose meter and to Medtronic Diabetes (Northridge, CA, USA) on their hospital-based continuous glucose monitor. CS-R declares that she has no competing interests.

\section{Author details}

'Division of Endocrinology, Boston Children's Hospital, 300 Longwood Avenue, Boston, MA 02115, USA. ${ }^{2}$ Division of Medicine Critical Care, Department of Medicine, Boston Children's Hospital; Department of Pediatrics, Harvard Medical School, 300 Longwood Avenue, Boston, MA 02115, USA.

Published: 14 June 2013

\section{References}

1. Finfer S, Wernerman J, Preiser JC, Cass T, Desaive T, Hovorka R, Joseph JI, Kosiborod M, Krinsley J, Mackenzie I, Mesotten D, Schultz MJ, Scott MG, Slingerland R, Van den Berghe G, Van Herpe T: Clinical review: Consensus recommendations on 'Measurement of blood glucose and reporting glycemic control in critically ill adults'. Crit Care 2013, 17:229.

2. Gijzen K, Moolenaar DL, Weusten JJ, Pluim HJ, Demir AY: Is there a suitable point-of-care glucose meter for tight glycemic control? Evaluation of one home-use and four hospital-use meters in an intensive care unit. Clin Chem Lab Med 2012, 50:1985-1992.

3. ISO 15197:2003: In vitro diagnostic test systems-requirements for bloodglucose monitoring systems for self-testing in managing diabetes mellitus. 2009-02-16

4. Heinemann L, Lodwig V, Freckmann G: Accuracy in blood glucose measurement: what will a tightening of requirements yield? J Diabetes Sci Technol 2012, 6:435-443.

5. ISO/FDIS 15197: In vitro diagnostic test systems - requirements for blood-glucose monitoring systems for self-testing in managing diabetes mellitus. Under development, stage 50.20.

6. Clinical and Laboratory Standards Institute: Point-of-Care Blood Glucose Testing in Acute and Chronic Care Facilities: Approved Guideline. 3rd edition. CLSI document POCT12-A3. Wayne, PA: Clinical and Laboratory Standards Institute; 2013

7. Agus MS, Steil GM, Wypij D, Costello JM, Laussen PC, Langer M, Alexander JL, Scoppettuolo LA, Pigula FA, Charpie JR, Ohye RG, Gaies MG; SPECS Study Investigators: Tight glycemic control versus standard care after pediatric cardiac surgery. N Engl J Med 2012, 367:1208-1219.

8. NICE-SUGAR Study Investigators, Finfer S, Chittock DR, Su SY, Blair D, Foster D, Dhingra V, Bellomo R, Cook D, Dodek P, Henderson WR, Hébert PC, Heritier S, Heyland DK, McArthur C, McDonald E, Mitchell I, Myburgh JA, Norton R, Potter J, Robinson BG, Ronco JJ: Intensive versus conventional glucose control in critically ill patients. N Engl J Med 2009, 360:1283-1297.

doi:10.1186/cc12599

Cite this article as: Soto-Rivera CL, Agus MSD: Measuring and reporting glycemic control in clinical trials: building a path to consensus. Critical Care 2013, 17:155 